\title{
Childhood wheezing phenotypes influence asthma, lung function and exhaled nitric oxide fraction in adolescence
}

\author{
Liesbeth Duijts $^{1,2,3}$, Raquel Granell ${ }^{1}$, Jonathan A.C. Sterne ${ }^{1}$ and \\ A. John Henderson ${ }^{1}$
}

Affiliations: 'School of Social and Community Medicine, University of Bristol, Bristol, UK. ${ }^{2}$ Dept of Pediatrics, Divisions of Respiratory Medicine and Neonatology, Erasmus University Medical Center, Rotterdam, The Netherlands. ${ }^{3}$ Dept of Epidemiology, Erasmus University Medical Center, Rotterdam, The Netherlands.

Correspondence: A. John Henderson, School of Social and Community Medicine, Oakfield House, Oakfield Grove, Bristol BS8 2BN, UK. E-mail: a.j.hendersonabris.ac.uk

ABSTRACT The objective of this study was to examine the associations of childhood wheezing phenotypes with asthma, lung function and exhaled nitric oxide fraction ( $F$ eNO) in adolescence.

In a population-based, prospective cohort study of 6841 children, we used latent class analysis to identify wheezing phenotypes during the first 7 years of life. Physician-diagnosed asthma, spirometry and FeNO were assessed at $14-15$ years.

Compared with never/infrequent wheeze, intermediate-onset and persistent wheeze were consistently strongest associated with higher risk of asthma (risk ratio (95\% CI) 10.9 (8.97-13.16) and 9.13 (7.74-10.77), respectively), lower forced expiratory volume in $1 \mathrm{~s}$ (FEV1)/forced vital capacity (FVC) ratio (mean difference in standard deviation units (SDU) $(95 \% \mathrm{CI})-0.34(-0.54--0.14)$ and $-0.50(-0.62--0.38)$, respectively), lower forced expiratory flow at $25-75 \%$ of FVC (FEF25-75\%) (mean difference in SDU (95\% CI) $-0.30(-0.49--0.10)$ and $-0.42(-0.54--0.30)$, respectively) and increased FEV 1 bronchodilator reversibility (mean difference in SDU (95\% CI) $0.12(0.02-0.22)$ and $0.13(0.06-0.19)$, respectively). Prolonged early and persistent wheeze were associated with a decline in FEV1/FVC ratio and FEF25-75\% between 8-9 and 14-15 years. Intermediate-onset, late-onset and persistent wheeze were associated with higher FeNO ratios (ratio of geometric means (95\% CI) 1.90 (1.59-2.29), 1.57 (1.39-1.77) and 1.37 (1.22-1.53), respectively, compared with never/infrequent wheeze).

Early-onset wheezing phenotypes persisting after 18 months of age show the strongest associations with asthma, lower lung function, even worsening from mid-childhood, and higher FeNO levels in adolescence.

$@$ ERSpublications

Early persisting wheezing phenotypes relate to asthma, lower lung function and higher FeNO levels in adolescence http://ow.ly/Su3xG

This article has supplementary material available from erj.ersjournals.com

Received: Aug 132013 | Accepted after revision: Aug 312015 | First published online: Dec 032015

Support statement: The UK Medical Research Council, the Wellcome Trust (grant reference 092731) and the University of Bristol provide core support for the Avon Longitudinal Study of Parents and Children. L. Duijts received a European Respiratory Society/Marie Curie Joint Research Fellowship (MC 1226-2009). The research leading to these results has received funding from the European Respiratory Society and the European Community's Seventh Framework Programme FP7/2007-2013 - Marie Curie Actions under grant agreement RESPIRE, PCOFUND-GA-2008-229571. R. Granell was supported by the UK Medical Research Council (grant number 0401540). Funding information for this article has been deposited with FundRef.

Conflict of interest: Disclosures can be found alongside the online version of this article at erj.ersjournals.com

Copyright (CERS 2016 


\section{Introduction}

Asthma is a complex heterogeneous disease comprising a number of discrete phenotypes. Childhood wheezing is the most common clinical manifestation of asthma onset. Severe and persistent childhood wheezing patterns are more likely to lead to asthma and abnormal pulmonary function in later life than mild and transient childhood wheezing patterns [1-4]. Recently, a novel symptom-driven approach to define different wheezing patterns was introduced using repeated measurements of wheeze during the first 7 years of childhood in a large population-based birth cohort study, the Avon Longitudinal Study of Parents and Children (ALSPAC) [5]. This led to the identification of six different phenotypes: never/ infrequent wheezing, transient early wheezing, prolonged early wheezing, intermediate-onset wheezing, late-onset wheezing and persistent wheezing. We found that intermediate-onset wheezing showed the strongest associations with atopy, airway hyperresponsiveness and lower lung function in mid-childhood. Thus far, it is not well understood whether the higher risk of asthma and abnormal pulmonary function in mid-childhood persists or worsen into adolescence, or whether different wheezing phenotypes in early childhood are related to eosinophilic airway inflammation, which can be assessed using the exhaled nitric oxide fraction $(\mathrm{FeNO})$

Therefore, we examined the associations of previously reported wheezing phenotypes during the first 7 years of childhood with asthma, lung function and FeNO in adolescence in the ALSPAC birth cohort study. We also examined whether any associations were modified by tobacco smoke exposure during pregnancy or early childhood and reported use of asthma medications.

\section{Methods}

Design and setting

This study was embedded in ALSPAC, a population-based birth cohort study of pregnant women resident in Avon, UK, and their children [6]. The cohort has been followed up with annual questionnaires and, since the age of 7 years, with objective measures in research clinics. The study protocol has been published previously [6] and further details can be found at http://www.bris.ac.uk/alspac. The study was conducted with the approval of the Avon Longitudinal Study of Parents and Children Ethics and Law Committee (IRB 00003312) and local research ethics committee, University Hospitals Bristol NHS Trust.

\section{Wheezing phenotypes}

At $6,18,30,42,54,69$ and 81 months after birth, mothers were sent a self-completion questionnaire about the health of their child. In two separate sections, they reported the occurrence of wheezing in the previous 12 months (6 months for the initial questionnaire) and, if present, whether they consulted a physician. Wheeze was defined as present if the response to any of the questions about wheezing was "yes" and absent if the response to both was "no". All other combinations were classified as missing (1.3\%).

\section{Asthma}

Information on asthma was obtained by a parent-completed questionnaire at age (mean \pm SD) $14 \pm 0.16$ years. Parents reported whether a physician ever diagnosed asthma or eczema in their child (no; yes, asthma; yes, eczema; yes, asthma and eczema) or whether their child had asthma during the past 12 months (no; yes, did not see a physician; yes, did see a physician). Based on the first obtained question, we grouped children into "physician-diagnosed asthma" (no; yes, asthma or asthma and eczema). Reported asthma during the past 12 months was used to reclassify physician-diagnosed asthma into "current physician-diagnosed asthma" (no; yes) where appropriate.

\section{Lung function}

Children visited research clinics at ages (mean \pm SD) $8.6 \pm 0.25$ and $15.4 \pm 0.30$ years. Lung function was measured by spirometry (Vitalograph 2120; Maids Moreton, UK) according to American Thoracic Society (ATS) criteria [7]. Each variable (forced expiratory volume in $1 \mathrm{~s} \mathrm{(FEV1),} \mathrm{forced} \mathrm{vital} \mathrm{capacity} \mathrm{(FVC)} \mathrm{and}$ forced expiratory flow at $25-75 \%$ of FVC (FEF25-75\%)) was converted into sex-, age- and height-adjusted standard deviation units (SDU) and percentage of predicted values of normal (online supplementary tables S4 and S5) [8]. Bronchodilator reversibility was calculated as the difference in SDU or percentage predicted lung function variables change before and $15 \mathrm{~min}$ after receiving $400 \mu \mathrm{g}$ salbutamol by metered aerosol and spacer. Lung function change between mid-childhood and adolescence was calculated as the difference in SDU or percentage predicted lung function variables between these two assessments.

\section{Fractional exhaled nitric oxide}

FeNO was measured online at a constant flow of $50 \mathrm{~mL} \cdot \mathrm{s}^{-1}$ according to European Respiratory Society/ATS guidelines using a Sievers NOA-280i nitric oxide analyser (GE Analytical Instruments, Boulder, CO, USA). Additionally, 758 children with less stringent criteria were included with individual blows $>5 \%$ deviation, 
but with at least one blow according to ATS criteria with adequate reach and duration of plateau, and FeNO value within an acceptable range of $10-200 \mathrm{ppb}$. Nonreproducibility was largely due to analyser dysfunction. We observed no difference in geometric means of FeNO values between the groups with and without children with less stringent criteria (geometric mean difference (95\% CI) $1.04(0.99-1.09) \mathrm{ppb}$ ), and no difference in size or direction of the effect estimates in our analyses when we included or excluded these children. FeNO measurements were done before spirometry measurements. Children were requested to omit their inhaled corticosteroids if applicable $48 \mathrm{~h}$ before their visit to the clinic. At the time of measurements, 14 children (0.3\%) received oral steroids, and 221 (5.2\%) had a chest infection and/or fever with a cold in the 3 weeks prior to the respiratory assessments. These children were excluded in our analyses as these factors are known to influence FeNO levels. As ambient nitric oxide concentrations could influence FeNO measurements, we excluded children with an extreme low or high nitric oxide with equivalent low or high ambient nitric oxide $(\mathrm{n}=1)$.

\section{Covariates}

Information on maternal age, deprivation index, anxiety, smoking during pregnancy and history of asthma or allergy were obtained from self-completion questionnaires at 18 or 32 weeks of gestation. Birthweight, gestational age, sex and ethnicity of the children were obtained from midwife and hospital registries at birth. Childhood maternal questionnaires from 3 to 15 months after birth provided information about breastfeeding, home overcrowding ( $>0.75$ persons per room), pet contact and childhood smoke exposure. At age 7 years, height was measured using a Harpenden stadiometer (Holtain, Crosswell, UK) and weight was measured using Tanita TBF 305 scales (Tanita, Viewsley, UK) from which body mass index (BMI) was calculated $\left(\mathrm{kg} \cdot \mathrm{m}^{-2}\right)$. At age 11 years, physical activity was measured by a MTI ActiGraph AM7164 2.2 accelerometer (ActiGraph, Pensacola, FL, USA) [9]. At age 14 years, parents reported if their child had a chest infection in the past 12 months, ever had hay fever or eczema and use of medication for asthma (no; yes, preventer inhaler; yes, reliever inhaler; yes, other inhaler or asthma medicine).

\section{Statistical analysis}

We used latent class analysis to derive distinct phenotypes of wheezing as described previously [5]. Briefly, this identifies the minimum number of classes that describe the variation of observed responses in multicategorical data, which in this case were wheeze at different time points [10]. The posterior probabilities (probability of each individual of belonging to each phenotype) were estimated, and used in generalised linear and weighted linear regression models to estimate associations of wheezing phenotypes with physician-diagnosed asthma, and lung function and FeNO levels, respectively, in adolescence. Linear regression models were used with bronchodilator reversibility and longitudinal lung function change between mid-childhood and adolescence as continuous outcome variables. Confounders were included in the adjusted models based on literature or change in effect estimates of $\geqslant 10 \%$. We additionally adjusted for lung function data in mid-childhood when lung function at age 14-15 years and changes between mid-childhood and adolescence were used as the outcomes. FeNO models were additionally adjusted for ambient nitric oxide. Data on confounders were missing for $7 \%$ at most and included in the analyses as separate categories or means. We explored effect modification of exposure to smoke during pregnancy or early life and use of asthma medication by including these variables as interaction terms in the linear regression models with lung function and FeNO as the outcomes. We tested the association between wheezing phenotypes and current wheezing severity at 8.6 years (number of wheezing/whistling attacks or asthma medication in the last 12 months: $0,1-2, \geqslant 3$ times) by ordered logistic regression (odds ratio (95\% CI) transient early $0.60(0.35-0.85)$, prolonged early 1.21 (0.98-1.45), intermediate-onset 3.93 (3.60-4.26), late-onset $2.72(2.51-2.94)$ and persistent wheezing $3.50(3.29-3.71))$. Owing to the strong observed associations and therefore the possibility of over-adjustment bias, we did not control our analyses for current wheezing severity. Finally, to assess whether the associations of the wheezing phenotypes with FeNO levels were modified by the use of inhaled corticosteroids, we repeated these analyses with FeNO levels as the outcome in strata of reported inhaled corticosteroid use (no, yes). FeNO levels were log-transformed to obtain normality, and therefore geometric means and ratios of geometric means are reported. Measures of associations with FeNO levels as the outcome are presented as back-transformed geometric means or ratios. All measures of association are presented with their $95 \%$ confidence intervals. Statistical analyses were performed using Stata version 11 (StataCorp, College Station, TX, USA).

\section{Results}

As previously analysed [5], wheezing phenotypes were characterised by timing of onset and subsequent decline or persistence: never/infrequent wheeze (no or low frequency wheeze $<6$ months; $61 \%$ ), transient early wheeze (6-18 months; 13\%), prolonged early wheeze (6-69 months; 10\%), intermediate-onset wheeze (>18 months; $2 \%$ ), late-onset wheeze (>42 months; $6 \%$ ) and persistent wheeze (>6 months; $8 \%$ ) [5]. 
Of 11678 children with information about wheezing phenotypes during the first 7 years of life, 6841 (59\%) had data on at least one respiratory outcome. Characteristics of the study population are shown in table 1.

\section{Wheezing phenotypes, asthma and lung function}

Compared with never/infrequent wheeze, all wheezing phenotypes were associated with asthma at age 15 years (online supplementary table S1). The strongest associations for asthma were observed for the intermediate-onset (relative risk ratio (95\% CI) 10.9 (8.97-13.16)) and persistent wheeze (relative risk ratio (95\% CI) 9.13 (7.74-10.77)) phenotypes, compared with never/infrequent wheeze.

Compared with never/infrequent wheeze in early childhood, only persistent wheeze was associated with lower levels of FEV1 (mean difference in SDU (95\% CI) $-0.14(-0.24--0.01)$ ) at age 15 years (figure 1a and online supplementary table S2). Wheezing phenotypes were associated with lower FEV1/FVC and, except for transient early wheeze, with lower FEF25-75\% (figure $1 \mathrm{~b}$ and c, respectively, and online supplementary table S2). Compared with never/infrequent wheeze, all wheezing phenotypes, except transient early or prolonged early wheeze, showed evidence of increased bronchodilator reversibility of FEV1 and FEF25-75\%, but not consistently for FEV1/FVC (figure $1 \mathrm{a}-\mathrm{c}$ and online supplementary table S2). The effect estimates of wheezing phenotypes with lung function in late childhood partly attenuated when analyses were additionally adjusted for respiratory outcomes in mid-childhood (online supplementary tables S2 and S3). Prolonged early and persistent wheeze were most consistently associated with a decline in change of FEV1/FVC ratio (mean difference in SDU (95\% CI) $-0.23(-0.34-0.13)$ and $-0.27(-0.39--0.15)$, respectively) and change of FEF25-75\% (mean difference in SDU (95\% CI) $-0.10(-0.20--0.00)$ and $-0.13(-0.24--0.02)$, respectively) between $8-9$ and $14-15$ years (figure $2 \mathrm{~b}$ and $\mathrm{c}$, and online supplementary table $\mathrm{S} 3$ ). We found no strong evidence for modification lung function by pre-natal smoke exposure, childhood smoke exposure or use of asthma medication (results not reported; p-values for interaction $>0.05$ ).

\section{TABLE 1 Characteristics of children and their mothers}

\begin{tabular}{|c|c|c|}
\hline & $\begin{array}{l}\text { With wheezing } \\
\text { phenotype data }\end{array}$ & $\begin{array}{l}\text { With wheezing phenotype and } \\
\text { respiratory assessments data }\end{array}$ \\
\hline Children & 11678 & 7278 \\
\hline \multicolumn{3}{|l|}{ Mother } \\
\hline Age at delivery years & $28.4 \pm 4.8$ & $29.1 \pm 4.6$ \\
\hline Maternal high education, $\mathrm{A}$-level or degree & $4131(35.4)$ & $3082(42.4)$ \\
\hline Parity $\geqslant 1$ & $6151(52.7)$ & $3731(51.3)$ \\
\hline $\begin{array}{l}\text { High anxiety index highest quartile at } \\
32 \text { weeks gestation }\end{array}$ & $1324(11.3)$ & 765 (10.5) \\
\hline Smoking during pregnancy & $2855(24.5)$ & $1374(18.9)$ \\
\hline History of asthma or allergy & $5057(43.3)$ & $3303(45.4)$ \\
\hline \multicolumn{3}{|l|}{ Child } \\
\hline Female & $5652(48.4)$ & $3643(50.1)$ \\
\hline Non-Caucasian ethnicity & $477(4.1)$ & $272(3.7)$ \\
\hline Pre-term birth $<37$ weeks & $686(5.9)$ & $373(5.1)$ \\
\hline Low birthweight $<2.5 \mathrm{~kg}$ & $579(5.0)$ & $328(4.5)$ \\
\hline Ever breastfed in first 6 months & $8278(70.9)$ & 5654 (77.7) \\
\hline Nursery during first year & $641(5.5)$ & $464(6.4)$ \\
\hline Pet contact during first year & $7442(63.7)$ & $4776(65.6)$ \\
\hline Childhood smoke exposure first year & $2494(21.4)$ & $1270(17.5)$ \\
\hline $\begin{array}{l}\text { High body mass index }>10 \text { th percentile } \\
\text { age } 7 \text { years }\end{array}$ & $748(6.4)$ & $583(8.0)$ \\
\hline $\begin{array}{l}\text { Low physical activity < } 10 \text { th percentile } \\
\text { age } 11 \text { years }\end{array}$ & $548(4.7)$ & $489(6.7)$ \\
\hline Lower respiratory tract infections age 14 years & $355(3.0)$ & $355(4.9)$ \\
\hline Age at respiratory assessments years & $15.5 \pm 0.33$ & $15.4 \pm 0.3$ \\
\hline Height age 15 years $\mathrm{cm}$ & $169.2 \pm 8.4$ & $169.3 \pm 8.4$ \\
\hline \multicolumn{3}{|l|}{ Asthma treatment age 14 years } \\
\hline "Preventer" inhaler & $425(3.6)$ & $424(5.8)$ \\
\hline "Reliever" inhaler & $609(5.2)$ & $604(8.3)$ \\
\hline Other inhaler or asthma medicine & $60(0.5)$ & $60(0.8)$ \\
\hline
\end{tabular}




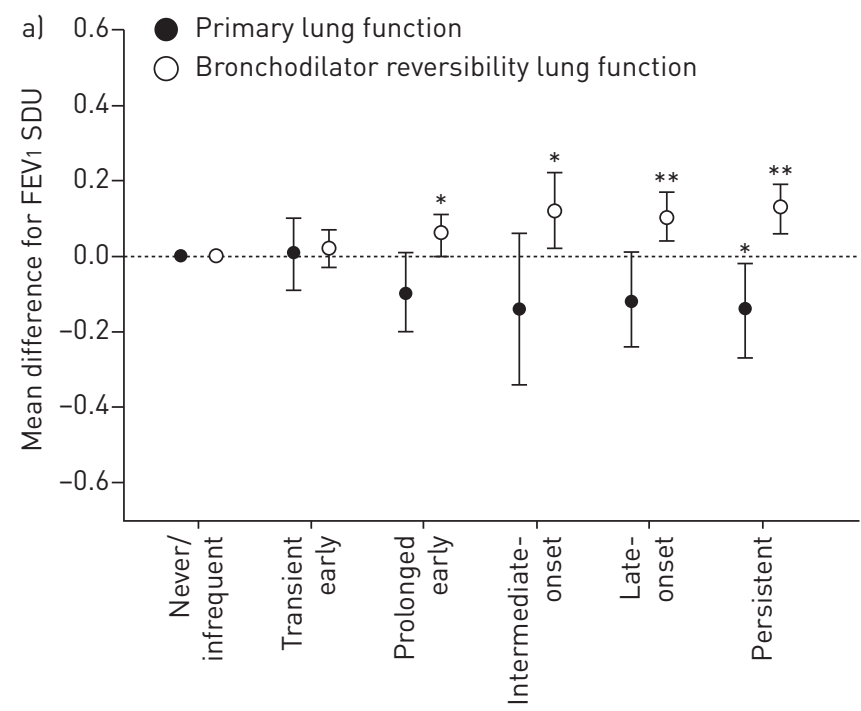

Wheezing phenotype

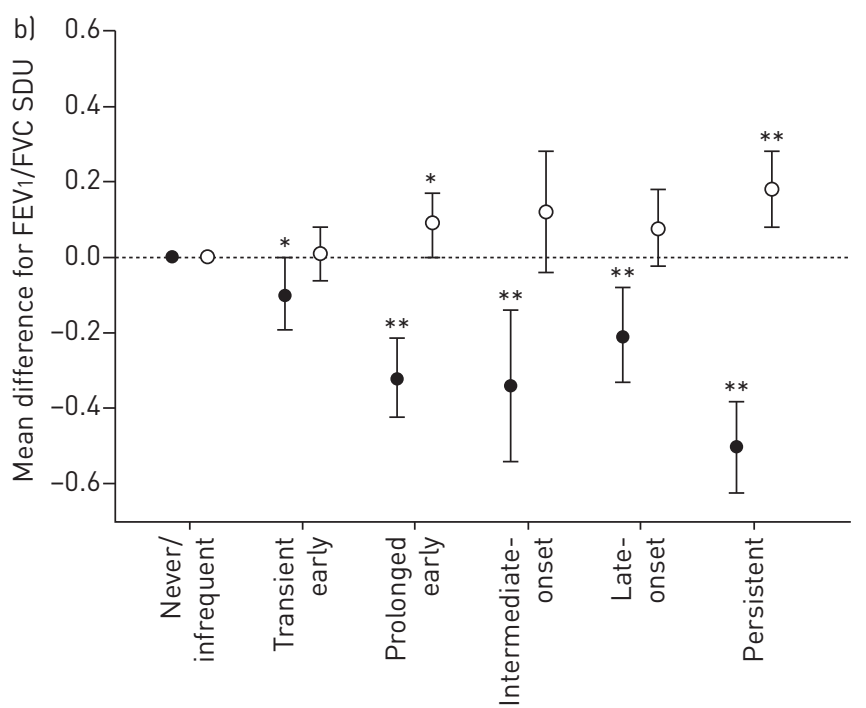

Wheezing phenotype

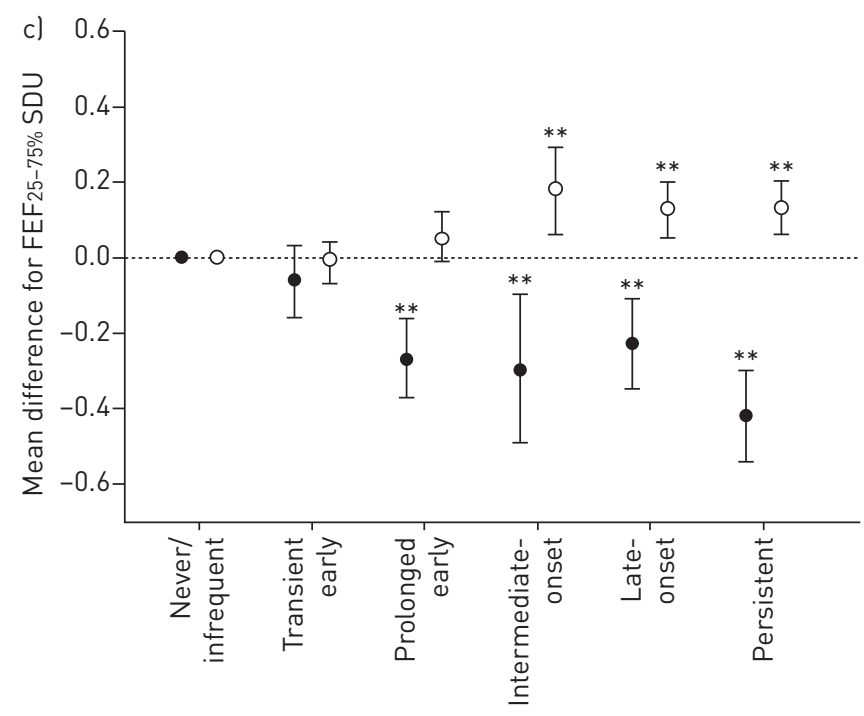

Wheezing phenotype

FIGURE 1 Association of wheezing phenotypes with lung function in adolescents (14-15 years). Data are presented as mean differences (95\% CI) of primary and bronchodilator reversibility lung function measurements compared with never/infrequent wheeze. a) Forced expiratory volume in $1 \mathrm{~s}(\mathrm{FEV} 1$ ). b) FEV 1 forced vital capacity (FVC). c) Forced expiratory flow at $25-75 \%$ of FVC (FEF25-75\%). Lung function values are presented as sex-, age- and height-adjusted standard deviation units (SDU). Models are adjusted for maternal age at delivery, education, parity, anxiety, smoking during pregnancy, history of asthma and allergy, children's sex, ethnicity, low birthweight, pre-term birth, breastfeeding, nursery, pet contact, childhood tobacco smoke exposure, high body mass index, low physical activity, and lower respiratory tract infections. Additional adjustment for lung function in mid-childhood is presented in online supplementary table S2. ${ }^{*}$ : $p<0.05 ;{ }^{* *}$ : $p<0.01$.

\section{Wheezing phenotypes and FeNO}

Compared with never/infrequent wheeze, intermediate-onset, late-onset and persistent wheeze were associated with higher ratios of $\mathrm{FeNO}$ at age $14-15$ years (ratio of geometric means (95\% CI) 1.90 (1.59-2.29), 1.57 (1.39-1.77) and 1.37 (1.22-1.53), respectively) (figure 3 and online supplementary table S4). Higher odds for FeNO were observed for these wheezing phenotypes when we used the 10th percentile cut-off level of high FeNO (OR (95\% CI) 1.34 (1.25-1.44), 1.20 (1.14-1.26) and 1.14 (1.09-1.20), respectively) (online supplementary table S7). Stratified analyses showed that the associations of intermediate-onset, late-onset and persistent wheeze with levels of FeNO were stronger in children who did not use a corticosteroid inhaler, but the evidence for an interaction was weak (online supplementary table S8; p-value for interaction $>0.05$ ). Similarly, there was no strong evidence that the associations of wheezing phenotypes with levels of FeNO were modified by smoke exposure during pregnancy or childhood smoke exposure (results not reported; p-values for interaction $>0.05$ ). 


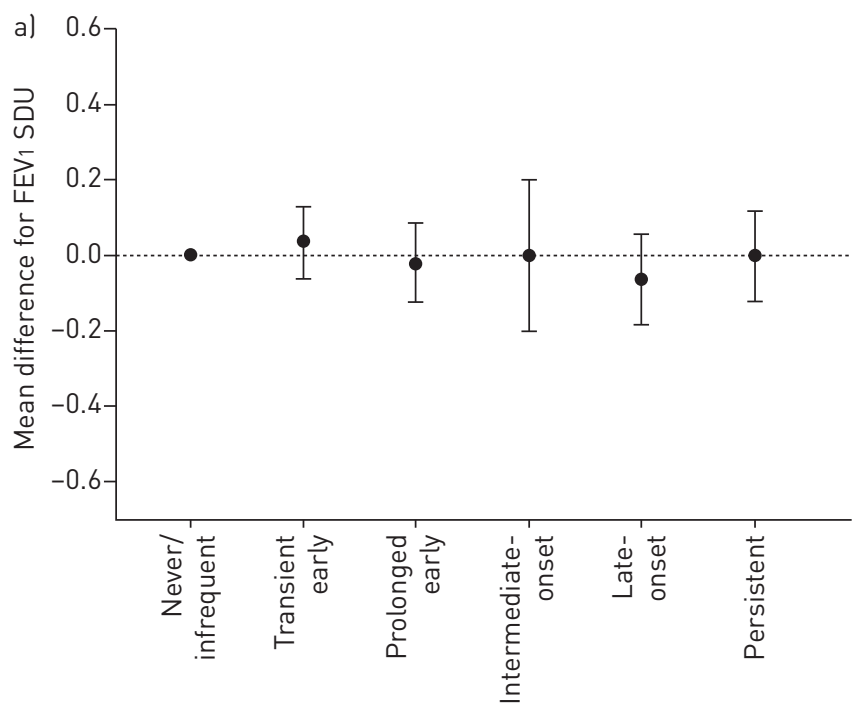

Wheezing phenotype

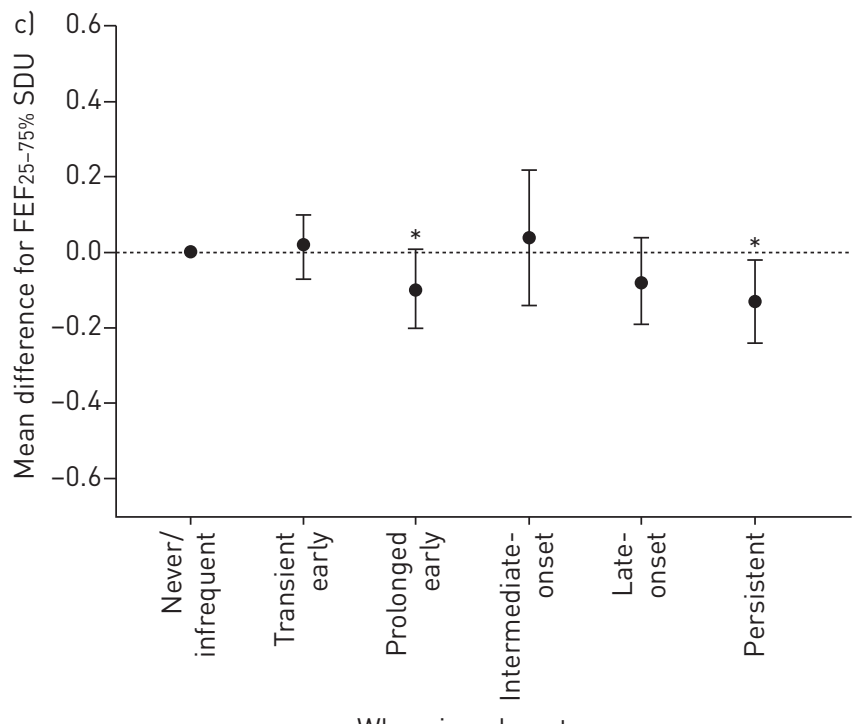

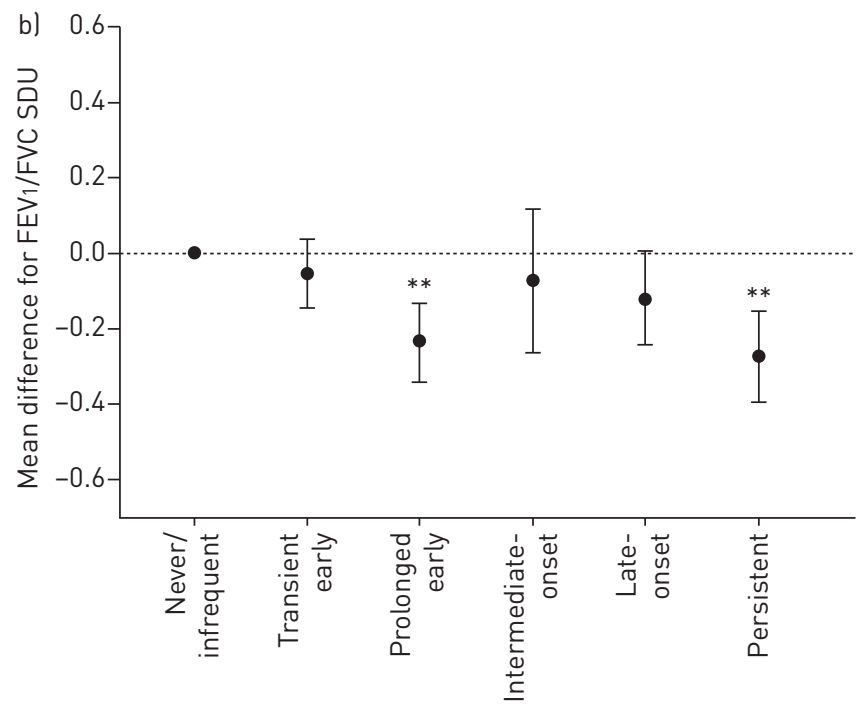

Wheezing phenotype

Wheezing phenotype

FIGURE 2 Association of wheezing phenotypes with lung function change between mid-childhood (8-9 years) and adolescence (14-15 years). Data are presented as mean differences $(95 \% \mathrm{Cl}$ ) compared with never/infrequent wheeze. a) Forced expiratory volume in $1 \mathrm{~s}$ (FEV1). b) FEV $1 /$ forced vital capacity (FVC). c) Forced expiratory flow at 25-75\% of FVC (FEF25-75\%). Lung function values are presented as sex-, age- and height-adjusted standard deviation units (SDU). Models are adjusted for maternal age at delivery, education, parity, anxiety, smoking during pregnancy, history of asthma and allergy, children's sex, ethnicity, low birthweight, pre-term birth, breastfeeding, nursery, pet contact, childhood tobacco smoke exposure, high body mass index, low physical activity, lower respiratory tract infections, and additionally for lung function in mid-childhood. ${ }^{*}: \mathrm{p}<0.05 ;{ }^{* *}$ : $\mathrm{p}<0.01$.

\section{Discussion}

Our results suggest that previously observed associations of wheezing phenotypes during the first 7 years of life with increased risk of asthma and lower lung function in mid-childhood persist until adolescence. Additionally, we observed that prolonged early and persistent wheeze were associated with a further decline in lung function between the ages of 8-9 and 14-15 years. Also, we found evidence for increased allergic airway inflammation at 14-15 years for the intermediate-onset, late-onset and persistent wheezing phenotypes.

\section{Interpretation of findings}

We previously identified two unreported wheezing phenotypes during the first 7 years of childhood: prolonged early and intermediate-onset wheezing [5]. The intermediate-onset wheezing phenotype was replicated [11] and validated [12] in two independent birth cohorts; additionally, evidence was provided that latent class analysis-derived wheezing phenotypes are clinically distinct and have different genetic origins $[13,14]$. We now show that the associations of intermediate-onset, late-onset and persistent wheezing phenotypes with asthma in adolescence are consistent with both our previously published 


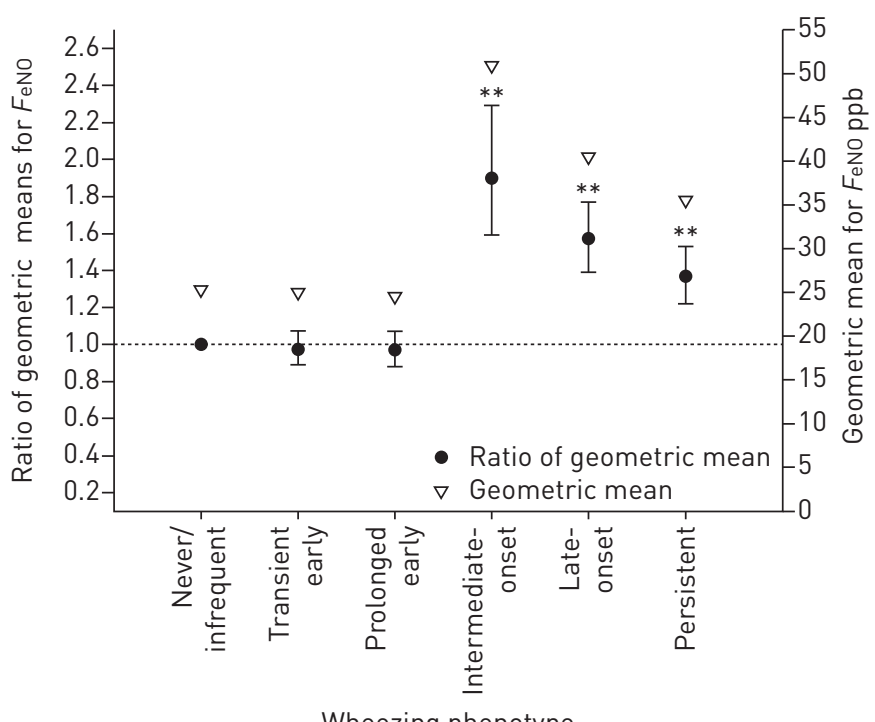

Wheezing phenotype

FIGURE 3 Association of wheezing phenotypes with fractional exhaled nitric oxide ( $\left.F_{\text {eNO}}\right)$ in adolescents (14-15 years). Data are presented as ratios of geometric means $(95 \% \mathrm{CI})$, as compared with never/infrequent wheeze, and geometric means. Models are adjusted for maternal age at delivery, education, parity, anxiety, smoking during pregnancy, history of asthma and allergy, children's sex, ethnicity, low birthweight, pre-term birth, breastfeeding, nursery, pet contact, childhood tobacco smoke exposure, high body mass index, low physical activity, lower respiratory tract infections, and ambient nitric oxide. ${ }^{* *}$ : $p<0.01$.

associations with outcomes at younger ages $[5,11]$ and other studies using different categories of wheezing and asthma in late childhood and adolescence [3,15]. Among currently wheezing children aged 10 years, asthma was diagnosed more often for persistent than for late-onset wheezing children $(76.0 \%$ versus $51.9 \%, \mathrm{p}<0.01$ ) [15]. Also, late-onset and persistent, and not transient early, wheezing children were more likely to continue to wheeze from age $8-9$ to $14-15$ years (OR (95\% CI) $3.12(2.5-3.9)$ and 3.8 (3.1-4.7), respectively) compared with children who never wheezed [3].

Previous studies reported inconsistent results for the associations of wheezing phenotypes with pulmonary function in adolescence [3, 15-19]. The differences in results might be explained by the different study designs, small number of subjects for sufficient power to detect small effects, definition of wheezing, time point and method of lung function measurement, e.g. spirometry, multiple-breath wash-out or peak flow variability. Of studies that observed associations between wheezing phenotypes and lung function measured by spirometry, the strongest and most consistent effects were observed for persistent wheezing with lower levels of $\mathrm{FEV}_{1}(-87$ to $-235 \mathrm{~mL})$ and $\mathrm{FEV}$ / $/ \mathrm{FVC}$ ratio $(-2.5 \%$ to $-3.4 \%)$ compared with the never wheezing phenotype $[3,15]$, even into adulthood $[2,18,19]$. Our results suggest that the two recently identified wheezing phenotypes, i.e. prolonged early and intermediate-onset, are also negatively associated with lung function into adolescence, in line with two recent published studies [18, 19]. Possible mechanisms of lower lung function in adolescence include developmental abnormalities persisting from infancy, and ongoing inflammation and repair of the airways leading to remodelling. This is supported by the evidence of increased airway inflammation levels in adolescence for our intermediate-onset, late-onset and persistent wheezing phenotypes.

We observed associations of prolonged early and persistent wheezing with change in lung function between 8-9 and 14-15 years, which implies that irreversible adverse changes persist and for some wheezing phenotypes further decline after early childhood. Our results are partly consistent with findings from the Tucson study that showed associations of early transient wheeze with lower maximal expiratory flow at the ages of 2.4 months and 6,11 and 16 years [3]. Late-onset wheeze was not associated with maximal expiratory flow at any age, but persistent wheeze was associated with maximal expiratory flow at 6 years and older. However, they did not observe a further decline of lung function after the age of 6 years for the phenotypes when compared with their peers. In contrast, and using different categories of wheezing or asthma phenotypes than this and our study, later studies observed that early persistent [18, 19], intermediate [19] and late-onset wheeze or asthma [18, 19] were associated with a reduced increase of FEV1 between 8-12 and 18 years compared with never/infrequent wheeze or asthma.

Our results with levels of FeNO as a respiratory morbidity outcome are not in line with previous studies, which did not show higher levels of FeNO due to a specific wheezing phenotype [17, 20-22]. Differences 
with our results could be explained by their use of other definitions of wheezing phenotype (multi-trigger versus episodic, atopic versus nonatopic, recurrent versus no wheezing phenotype), partly lack of adjustment for confounders, younger age at which FeNO levels were measured and smaller sample-sized cohorts. Our observed effect estimates of the associations between wheezing phenotypes and FeNO were consistently strongest for intermediate-onset wheeze when mean levels of FeNO, ratios of continuously measured FeNO or 10th percentile cut-off levels of FeNO were used. This might be due to different specific underlying mechanisms for specific wheezing phenotypes. We speculate that the intermediate-onset and late-onset wheezing phenotypes mostly arise due to atopic mechanisms including sensitivity to common allergens [5, 23], leading to higher risk of (atopic) asthma, higher levels of FeNO and lower lung function, while the persistent wheezing phenotype arises due to a mixture of growth, infectious and atopic mechanisms [24-27], leading to lower lung function only. FeNO levels were low compared with clinic-based samples. However, in the current population-based sample, which probably contains a higher proportion of mild asthmatics, there was still clear evidence of wheezing phenotype-associated airway inflammation. Although controlling our analyses for current wheezing severity could have led to over-adjustment bias, we cannot rule out that part of the observed associations is explained by current wheezing severity.

\section{Strengths and methodological issues}

This study was embedded in a population-based, prospective cohort study with a large number of subjects being studied from early life onwards, with detailed and frequently measured information about wheezing, asthma and lung function, and adjustment for a large number of confounders. Furthermore, this cohort study is the first study that measured levels of FeNO in adolescence. In common with most longitudinal cohort studies, there was loss to follow-up. Of children with complete cases on wheezing until age 7 years, we had substantial missing values on respiratory assessments at age 15 years. However, imputing missing of respiratory outcome data would have led to bias because of the not-at-random nature of the missing data [28]. Selection bias due to loss to follow-up would be present in our study if the associations of wheezing phenotypes with respiratory outcomes were different between adolescents lost to follow-up and those who remained in the study. Although this cannot be totally excluded, qualitative conclusions about the direction and approximate magnitude of the effect estimates will most probably not change [29]. We used parental-reported wheezing symptoms, which is widely accepted in epidemiological studies [30]. Under- or over-reporting could be present, but misclassification of wheeze influencing our results seems unlikely because seven observations were used and latent class analyses allows for misclassification. However, data on treatment with inhaled steroids was not available before 8 years of age and misclassification due to suppression of symptoms by treatment might be present. Additionally, misclassification due to over- or under-reporting or reduced compliance of use of inhaled steroids at age 14-15 years might have been present. Although the interaction was weak, we observed stronger associations of wheezing phenotypes with FeNO for those who did not use inhaled corticosteroid than those who did. This might be due to declining FeNO levels due to treatment. The geometric mean of FeNO for the never/infrequent wheeze group was high, but should be interpreted carefully due to statistical transformation. The wheeze patterns that were observed in our data were not constrained by pre-specified notions of their number or nature. The strong associations of the wheezing phenotypes with asthma, lung function and levels of FeNO until adolescence confirms the appropriate use of wheeze patterns derived by latent class analyses at least in population-based cohorts studying underlying mechanisms and prognosis of respiratory morbidity. Other phenotypes such as episodic viral and multi-trigger wheeze might be more appropriate for clinical practice [31], although clinical phenotypes seem well supported by latent cluster analysis [32]. Which identified classifications of wheeze [5, 18, 19, 33], or classifications of diverse inflammatory markers and symptoms $[34,35]$, eventually merge so that underlying pathology or diseases are the best reflected remains to be studied. Still, our results, including that specific wheezing phenotypes in early life lead to lower lung function, and even worsening between mid- and late childhood, and increased airway inflammation, could be used by healthcare workers to develop preventive screening strategies to optimise the early phase of life to prevent symptoms and disease in the long term. We did not control for asthma severity at younger ages, which is suggested to be a predictor for disease persistence or progression at older ages, to avoid the possibility of over-adjustment. Although we adjusted for a large number of confounders, residual confounding due to early life respiratory tract infections or genetic variants [36-38] might be present.

\section{Conclusion}

Our study suggests that associations of wheezing patterns in early childhood with increased odds of asthma and lower lung function in mid-childhood persist until adolescence. Lung function from mid-childhood until adolescence further declined for the prolonged early and persistent wheezing phenotypes. We found evidence for increased allergic airway inflammation in adolescence for wheezing phenotypes with early 
onset that persisted after 18 months of age. Further studies are needed to explore possible underlying mechanisms to develop specific intervention strategies that optimise lung development in early life and prevent additional decline by adolescence.

\section{Acknowledgements}

We are extremely grateful to all the families who took part in this study, the midwives for their help in recruiting them, and the whole Avon Longitudinal Study of Parents and Children (ALSPAC) team, which includes interviewers, computer and laboratory technicians, clerical workers, research scientists, volunteers, managers, receptionists, and nurses. We thank H.T. den Dekker (Dept of Pediatrics, Division of Respiratory Medicine and Dept of Epidemiology, Erasmus University Medical Center, Rotterdam, The Netherlands) for creating the figures.

\section{References}

1 Jenkins MA, Hopper JL, Bowes G, et al. Factors in childhood as predictors of asthma in adult life. BMJ 1994; 309 : 90-93.

2 Sears MR, Greene JM, Willan AR, et al. A longitudinal, population-based, cohort study of childhood asthma followed to adulthood. N Engl J Med 2003; 349: 1414-1422.

3 Morgan WJ, Stern DA, Sherrill DL, et al. Outcome of asthma and wheezing in the first 6 years of life: follow-up through adolescence. Am J Respir Crit Care Med 2005; 172: 1253-1258.

4 Phelan PD, Robertson CF, Olinsky A. The Melbourne Asthma Study: 1964-1999. J Allergy Clin Immunol 2002; 109: 189-194.

5 Henderson J, Granell R, Heron J, et al. Associations of wheezing phenotypes in the first 6 years of life with atopy, lung function and airway responsiveness in mid-childhood. Thorax 2008; 63: 974-980.

6 Boyd A, Golding J, Macleod J, et al. Cohort profile: the 'children of the 90s' - the index offspring of the Avon Longitudinal Study of Parents and Children. Int J Epidemiol 2012; 42: 111-127.

7 American Thoracic Society. Standardization of spirometry, 1994 update. Am J Respir Crit Care Med 1995; 152: $1107-1136$.

8 Quanjer PH, Stanojevic S, Cole TJ, et al. Multi-ethnic reference values for spirometry for the 3-95-yr age range: the global lung function 2012 equations. Eur Respir J 2012; 40: 1324-1343.

9 Ness AR, Leary SD, Mattocks C, et al. Objectively measured physical activity and fat mass in a large cohort of children. PLoS Med 2007; 4: e97.

10 Rabe-Hesketh S, Skrondal A. Classical latent variable models for medical research. Stat Methods Med Res 2008; 17: 5-32.

11 Savenije OE, Granell R, Caudri D, et al. Comparison of childhood wheezing phenotypes in 2 birth cohorts: ALSPAC and PIAMA. J Allergy Clin Immunol 2011; 127: 1505-1512.

12 Collins SA, Pike KC, Inskip HM, et al. Validation of novel wheeze phenotypes using longitudinal airway function and atopic sensitization data in the first 6 years of life: evidence from the Southampton Women's survey. Pediatr Pulmonol 2013; 48: 683-692.

13 Spycher BD, Henderson J, Granell R, et al. Genome-wide prediction of childhood asthma and related phenotypes in a longitudinal birth cohort. J Allergy Clin Immunol 2012; 130: 503-509.

14 Granell R, Henderson AJ, Timpson N, et al. Examination of the relationship between variation at $17 \mathrm{q} 21$ and childhood wheeze phenotypes. J Allergy Clin Immunol 2013; 131: 685-694.

15 Kurukulaaratchy RJ, Fenn MH, Waterhouse LM, et al. Characterization of wheezing phenotypes in the first 10 years of life. Clin Exp Allergy 2003; 33: 573-578.

16 Stein RT, Holberg CJ, Morgan WJ, et al. Peak flow variability, methacholine responsiveness and atopy as markers for detecting different wheezing phenotypes in childhood. Thorax 1997; 52: 946-952.

17 Sonnappa S, Bastardo CM, Wade A, et al. Symptom-pattern phenotype and pulmonary function in preschool wheezers. J Allergy Clin Immunol 2010; 126: 519-526.

18 Hallberg J, Thunqvist P, Schultz ES, et al. Asthma phenotypes and lung function up to 16 years of age - the BAMSE cohort. Allergy 2015; 70: 667-673.

19 Lodge CJ, Lowe AJ, Allen KJ, et al. Childhood wheeze phenotypes show less than expected growth in $\mathrm{FEV}_{1}$ across adolescence. Am J Respir Crit Care Med 2014; 189: 1351-1358.

20 Moeller A, Diefenbacher C, Lehmann A, et al. Exhaled nitric oxide distinguishes between subgroups of preschool children with respiratory symptoms. J Allergy Clin Immunol 2008; 121: 705-709.

21 Kappelle L, Brand PL. Severe episodic viral wheeze in preschool children: high risk of asthma at age 5-10 years. Eur J Pediatr 2012; 171: 947-954.

22 Brussee JE, Smit HA, Kerkhof M, et al. Exhaled nitric oxide in 4-year-old children: relationship with asthma and atopy. Eur Respir J 2005; 25: 455-461.

23 Scott M, Raza A, Karmaus W, et al. Influence of atopy and asthma on exhaled nitric oxide in an unselected birth cohort study. Thorax 2010; 65: 258-262.

24 Sonnenschein-van der Voort AM, Jaddoe VW, Raat H, et al. Fetal and infant growth and asthma symptoms in preschool children. The Generation R Study. Am J Respir Crit Care Med 2012; 185: 731-737.

25 Kotecha SJ, Watkins WJ, Paranjothy S, et al. Effect of late preterm birth on longitudinal lung spirometry in school age children and adolescents. Thorax 2012; 67: 54-61.

26 Shaheen SO, Sterne JA, Tucker JS, et al. Birth weight, childhood lower respiratory tract infection, and adult lung function. Thorax 1998; 53: 549-553.

27 Turner S, Prabhu N, Danielan P, et al. First- and second-trimester fetal size and asthma outcomes at age 10 years. Am J Respir Crit Care Med 2011; 184: 407-413.

28 White IR, Carlin JB. Bias and efficiency of multiple imputation compared with complete-case analysis for missing covariate values. Stat Med 2010; 29: 2920-2931.

29 Howe LD, Tilling K, Galobardes B, et al. Loss to follow-up in cohort studies: bias in estimates of socioeconomic inequalities. Epidemiology 2013; 24: 1-9.

30 Jenkins MA, Clarke JR, Carlin JB, et al. Validation of questionnaire and bronchial hyperresponsiveness against respiratory physician assessment in the diagnosis of asthma. Int J Epidemiol 1996; 25: 609-616. 
31 Brand PL, Baraldi E, Bisgaard H, et al. Definition, assessment and treatment of wheezing disorders in preschool children: an evidence-based approach. Eur Respir J 2008; 32: 1096-1110.

32 Depner M, Fuchs O, Genuneit J, et al. Clinical and epidemiologic phenotypes of childhood asthma. Am J Respir Crit Care Med 2014; 189: 129-138.

33 Spycher BD, Silverman M, Brooke AM, et al. Distinguishing phenotypes of childhood wheeze and cough using latent class analysis. Eur Respir J 2008; 31: 974-981.

34 Just J, Gouvis-Echraghi R, Rouve S, et al. Two novel, severe asthma phenotypes identified during childhood using a clustering approach. Eur Respir J 2012; 40: 55-60.

35 Clarisse B, Demattei C, Nikasinovic L, et al. Bronchial obstructive phenotypes in the first year of life among Paris birth cohort infants. Pediatr Allergy Immunol 2009; 20: 126-133.

36 Moffatt MF, Kabesch M, Liang L, et al. Genetic variants regulating ORMDL3 expression contribute to the risk of childhood asthma. Nature 2007; 448: 470-473.

37 Hancock DB, Eijgelsheim M, Wilk JB, et al. Meta-analyses of genome-wide association studies identify multiple loci associated with pulmonary function. Nat Genet 2010; 42: 45-52.

38 Repapi E, Sayers I, Wain LV, et al. Genome-wide association study identifies five loci associated with lung function. Nat Genet 2010; 42: 36-44. 